\section{§39. Anisotropic Pressure Effects on Plasma Equilibrium in Toroidal Systems}

Pustovitov, V.D. (Institute of Tokamak Physics, Russian Research Centre "Kurchatov Institute")

General properties of anisotropic plasma equilibrium in tokamaks and stellarators are considered within the approach allowing explicit analytical evaluation of the equilibrium plasma currents and the related integral quantities. This is done to provide a link to conventional equilibrium theory for isotropic plasma, find the rules for possible extensions of its results to configurations with anisotropic pressure, and to unify the knowledge obtained in separate numerical studies. Expressions for the diamagnetic signal and, mainly, the Pfirsch-Schlüter current in stellarators are considered. Ultimately this goes to theoretical justification of the method of experimental evaluation of the pressure anisotropy in the Large Helical Device (LHD) proposed in [1]. The conditions of its applicability are clarified and possible limitations are elucidated. Combined effects of the pressure anisotropy and inward-outward shift of the plasma on the Pfirsch-Schlüter current in stellarators are discussed.

The analysis is based on the equation

$$
\nabla \cdot \vec{p}=\mathbf{j} \times \mathbf{B},
$$

where $\mathbf{j}$ is the current density, $\mathbf{B}$ is the magnetic field,

$$
\vec{p}=p_{\|} \frac{\mathbf{B B}}{\mathbf{B}^{2}}+p_{\perp}\left(\overrightarrow{\mathbf{I}}-\frac{\mathbf{B B}}{\mathbf{B}^{2}}\right),
$$

$p_{\|}$and $p_{\perp}$ are the parallel and perpendicular pressures, and $\overrightarrow{\mathbf{I}}$ is the unit dyadic. This equation is solved analytically to find the equilibrium current density.

With poloidal current found from (1), the diamagnetic signal $\Delta \Phi$ is calculated in the cylindrical approximation, but with account of the helical magnetic field. The result for stellarators with anisotropic plasma and, possibly, not too large toroidal current is (compare to [2])

$$
2 \frac{\Delta \Phi}{\Phi_{0}}=\frac{B_{J}^{2}}{B_{0}^{2}}-\bar{\beta}_{\perp}+2 \frac{\Delta \Phi_{s t}}{\Phi_{0}},
$$

where $\Phi_{0}=B_{0} S$ with $S=\pi b^{2}$ the cross-section of the plasma, $b$ is its minor radius, $B_{0}$ is the toroidal field, $B_{J}$ is the poloidal field at the plasma boundary due to the net toroidal current, $\bar{\beta}_{\perp} \equiv 2 \bar{p}_{\perp} / B_{0}^{2}$ is the ratio of the volume-averaged plasma perpendicular pressure to the magnetic field pressure $B_{0}^{2} / 2$, and $\Delta \Phi_{s t}$ is a term due to the combined effect of the stellarator helical field and the toroidal current. Detailed derivations of isotropic version of $\Delta \Phi_{s t}$ for a stellarator are described in [3]. This term is determined by the profiles of the toroidal current density and the stellarator rotational transform [3], and the plasma anisotropy does not affect this dependence.

It is shown that the parallel force balance leads to

$$
p_{\|}=p_{\| 0}+\widetilde{p}_{\|}
$$

with

$$
\widetilde{p}_{\|}=0.5\left(p_{\| 0}-p_{\perp}\right)\left(1-\mathbf{B}_{0}^{2} / \mathbf{B}^{2}\right) .
$$

Here the surface function $p_{\| 0}=p_{\| 0}(a)$ is $p_{\|}$at the point where $\mathbf{B}^{2}=\mathbf{B}_{0}^{2}$. This shows that $\widetilde{p}_{\|}$must be small even at significant variation of $p_{\perp}$ around a flux surface.

With this property the Pfirsch-Schlüter current is calculated. This is needed for calculating the signals measured by the saddle coils [1]. For isotropic plasma this current is approximately given by [4]

$$
j_{P S}=R p^{\prime}(\psi)\left(\Omega-\left\langle\Omega_{0}\right\rangle\right),
$$

where $R$ is the major radius, $\psi$ is the poloidal flux,

$$
\Omega=1-\frac{R^{2}}{r^{2}}+\frac{\left\langle\widetilde{\mathbf{B}}^{2}\right\rangle_{\zeta}}{B_{0}^{2}},
$$

$r$ is the radius from the main axis, and the last term describes the "magnetic hill" effect. The analysis show that, for anisotropic plasma with weakly inhomogeneous $p_{\perp}$, expression (6) remain valid if $p$ is replaced by

$$
p=0.5\left(p_{\| 0}+p_{\perp 0}\right)
$$

where $p_{\perp 0}$ is the averaged $p_{\perp}$. Expression (6) with (8) allows easy generalization of the conventional MHD equilibrium theory results on the case of anisotropic plasma. It confirms the main assumptions accepted in [1].

If $p_{\perp}$ is near a surface function, which may or may not be in experiments, then the expressions for the Shafranov shift and the related quantities obtained for isotropic plasma are simply modified by the replacement (8). This was known for some special cases. Now we extend this for wider class of tokamaks and stellarators.

Strong departure of $p_{\perp}$ from homogeneous $p_{\perp 0}(a)$ makes (6) less accurate and results in an additional contributions to $j_{P S}$ with higher harmonics than the main dipole component, which must be present equally in tokamaks and stellarators.

For stellarators, analysis predicts one more possible contribution to the main harmonic of $j_{P S}$. Its amplitude depends on the poloidal variation of $p_{\perp}$ on magnetic surfaces and a shift of these surfaces with respect to the "standard" configuration. Combination of these two factors can, probably, engender a detectable effect. This might be of interest for future experiments in LHD with a new perpendicular NBI of $7 \mathrm{MW}$ [5].

For complete derivations and discussions see [6].

[1] T. Yamaguchi et al., Nucl. Fusion, 45, L33 (2005).

[2] T. Yamaguchi et al., Plasma Phys. Control. Fusion, 48, L73 (2006).

[3] V.D. Pustovitov and V.D. Shafranov, Reviews of Plasma Physics, ed. B.B. Kadomtsev (New York: Consultants Bureau), 1990, Vol. 15, pp. 163-326.

[4] V.D. Pustovitov, Nucl. Fusion, 36, 583 (1996).

[5] A. Komori et al., Nucl. Fusion, 49, 104015 (2009).

[6] V.D. Pustovitov, Plasma Phys. Control. Fusion, 52, 065001 (2010). 